\title{
Vài con số hoạt động NCKH Phenikaa Uni qua dữ liệu Scopus
}

\author{
Hồ Mạnh Toàn \\ Trung tâm ISR
}

Hà Nội, 11-9-2020

URL: http://isr.phenikaa-uni.edu.vn/chitiet/tin-tuc 2019222758 84/vai-con-so-hoat-dong-nckhphenikaa-uni-qua-du-lieu-scopus

Nghiên cứu khoa học (NCKH) là một trọng tâm của trường Đại học Phenikaa trong quá trình hướng tới mục tiêu trở thành trường đại học nghiên cứu có uy tín quốc tế.

Bên cạnh hệ thống dữ liệu của ISI Web of Science-hiện nay phần nào có thể tra cứu được qua Publons - thì Scopus đang ngày càng đóng vai trò quan trọng. Đặc biệt, nhiều bảng xếp hạng đại học toàn cầu, mà gần đây nhất là Times Higher Education World University Rankings (THE-WUR), đang hợp tác sử dụng dữ liệu Scopus và thuật toán SciVal. Đồng thời, SCImago Lab cũng sử dụng dữ liệu Scopus, và nhờ tra cứu rộng rãi, tiện dụng SCImago ngày càng trở nên quen thuộc và hữu dụng trong tìm kiếm thông tin khoa học $[1,2]$.

Bài SciComm ngắn này không đặt ra mục tiêu trình bày đầy đủ về dữ liệu Scopus của Phenikaa Uni, một phần vì đây là dữ liệu bản quyền. Bài chỉ nhằm điểm vài nét cơ bản về Phenikaa Uni qua những gì Scopus cung cấp, cụ thể bằng vài hình ảnh đơn giản [1].

\section{Affiliation details - Phenikaa University}

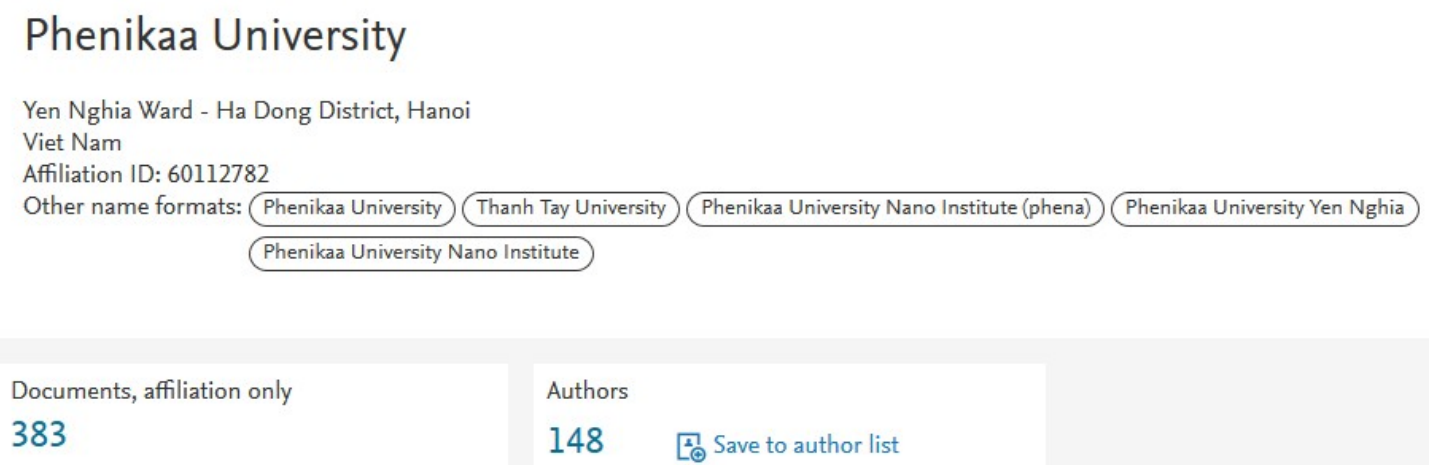


https://www.scopus.com/affil/profile.uri?afid=60112782

Mã số định danh Phenikaa University trong hệ thống Scopus (Affiliation ID) là: 60112782. Khi thống kê dữ liệu khoa học cho Phenikaa Uni, Scopus thống kê cả dữ liệu kế thừa với tên gọi cũ "Thanh Tay University" và các viện trực thuộc, theo vài phiên bản tên đã nhận dạng được như trong hình.

Scopus bắt đầu có dữ liệu xuất bản khoa học đầu tiên của Phenikaa Uni từ năm 2017. Những dòng dữ liệu đầu tiên của 2021 cũng đã sớm xuất hiện (do cách đánh số volume và issue của từng ấn phẩm).

Một điều rất thú vị là Phenikaa Uni đã bắt nhịp với xu hướng hiện đại, đổi mới cởi mở toàn cầu về "Open Science". Biểu hiện cụ thể là tỷ trọng nội dung truy cập mở (OA) của trường là: 157 (trên tổng số 383). Như chúng ta đã biết, với phong trào khoa học Plan S, Open Science, và đòi hỏi chia sẻ tài nguyên khoa học bắt buộc đang nóng bỏng khắp thế giới, các nhà tài trợ khoa học lớn như NSF (Mỹ), ERC (EU), Wellcome Trust (Anh), Bill \& Melinda Gates Foundation (Mỹ), v.v.. đang bắt buộc tất cả các nhà khoa học muốn nhận được hỗ trợ tài chính đều phải tuân thủ các điều kiện công bố truy cập mở và lưu trữ mở để phục vụ nhu cầu nhân loại.

Trong hình ảnh dưới đây, các lĩnh vực mà các nhà khoa học Phenikaa Uni tham gia đóng góp nhiều nhất là: 1) Khoa học vật liệu; 2) Vật lý và thiên văn; 3) Kỹ thuật; 4) Hóa học.

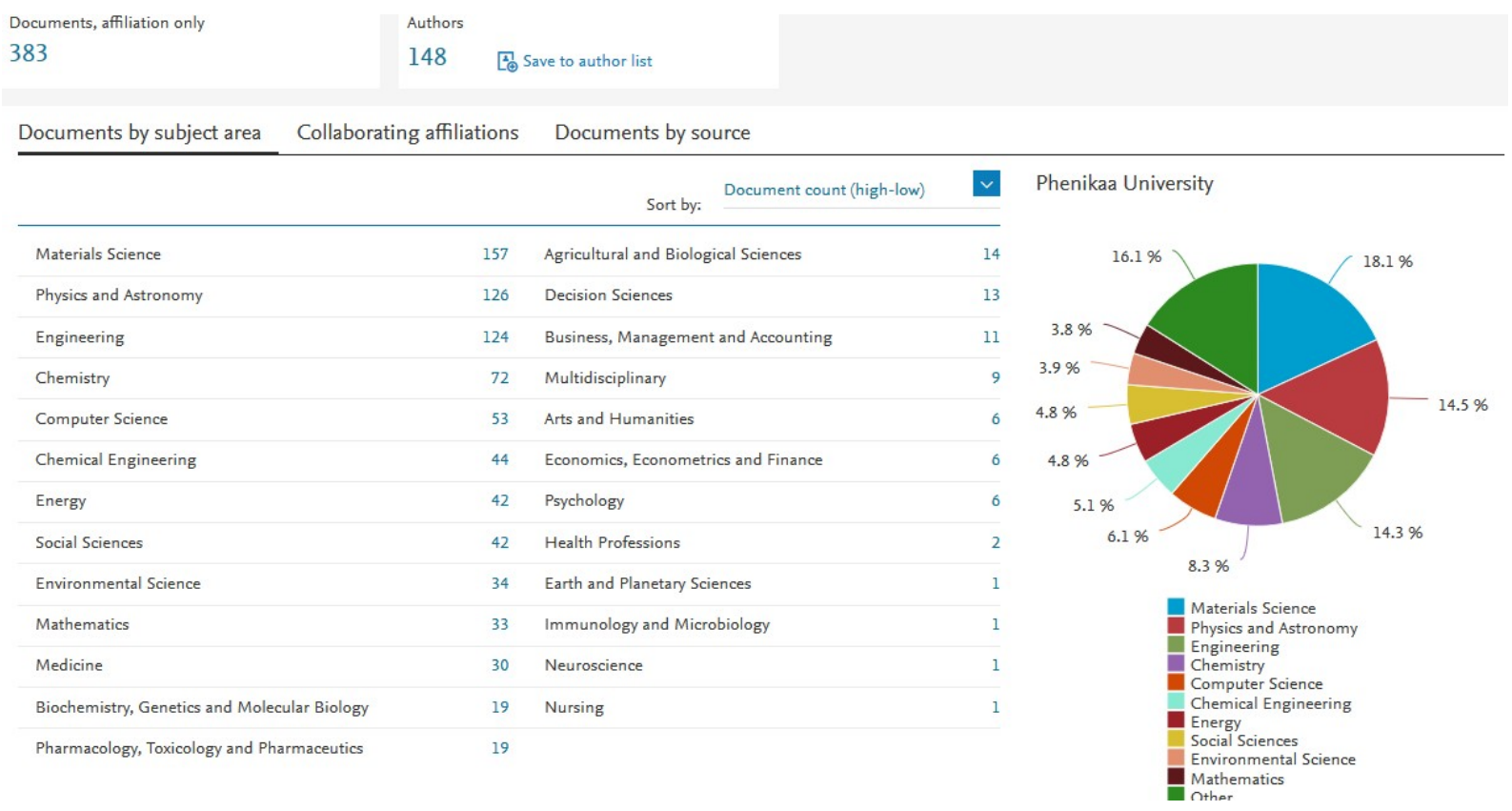

Tỷ trọng đóng góp NCKH theo ngành

Tổng số tác giả Phenikaa Uni tương đương với ISI Web of Science, 75, đó là do phần đông các ấn phẩm chỉ mục hóa trong Scopus thì cũng có mặt trong WOS. Tuy nhiên, trong Scopus do việc thiết kế và thực 
hành lưu trữ dữ liệu, một số bản ghi tách rời và chưa được hợp nhất dữ liệu, do đó thống kê là 148. (Vấn đề sai lệch thống kê này của các hệ thống bibliographic database đã được nêu rõ trong nghiên cứu [3].)

Tiếp theo, hình ảnh bên dưới cho biết một ví dụ về một số tác giả có đóng góp tiêu biểu trong Scopus, đang công tác tại Phenikaa Uni. Dẫn đầu danh sách là GS. Nguyễn Văn Hiếu với 143 bài xuất bản.

(Cũng xin lưu ý là do cùng vấn đề về tính "bảo thủ" đã trình bày trong [3], con số này đang thấp hơn đóng góp thực tế của GS. Hiếu. Sai số có thể được hiệu chỉnh, nhưng mất nhiều thời gian. Do đó, có thể nói dữ liệu thống kê của Scopus và Web of Science cơ bản có tính "bảo thủ", cũng theo [4].)

Lượng trích dẫn tổng cộng của GS. Hiếu cũng dẫn đầu và rất lớn: 3612, tiếp theo sau là tác giả Raja Das: 2238 (tính đến 10-9-2020).

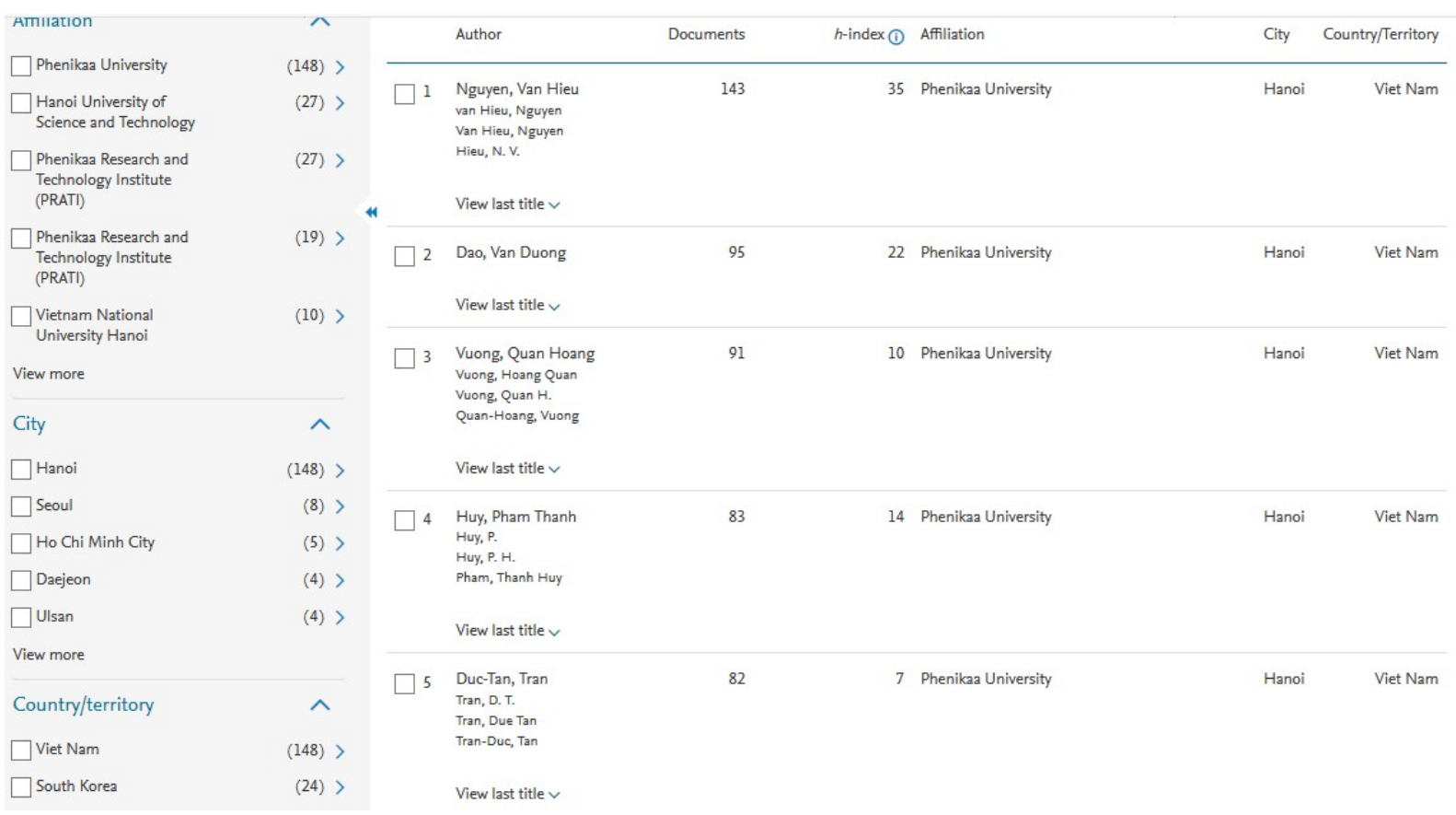

Ví dụ một số tác giả Phenikaa Uni trong Scopus

Một điểm đáng chú ý nữa là, GS. Hiệu trưởng Phạm Thành Huy cũng là một tác giả đóng góp dẫn đầu trong danh sách Scopus của Phenikaa Uni.

Một khía cạnh khác rất quan trọng của công việc NCKH là ảnh hưởng của sản phẩm khoa học. Trên thực tế, có vài cách hiểu bổ sung qua lại cho nhau về thế nào là ảnh hưởng của sản phẩm NCKH. Tuy vậy, cách phổ biến trong giới học thuật là thông qua lượng trích dẫn.

Về mặt này, cũng tương tự ISI Web of Science, các bài xuất bản dưới tên Phenikaa Uni trong hệ thống Scopus đều được thống kê lượt trích dẫn ("citation" hoặc "times cited"). 
(Ta cũng lưu ý là lượng trích dẫn này được đếm theo cách cũng "bảo thủ", nghĩa là chỉ khi nào một bài chỉ mục hóa trong Scopus trích dẫn một bài khác cũng trong Scopus thì mới xuất hiện 1 citation được đếm. Cách hiểu bảo thủ này cũng có nhiều hạn chế, ví dụ trích dẫn trong các ấn phẩm rất quan trọng, chẳng hạn luận án tiến sỹ, lại không được tính.)

Hình ảnh dưới đây là một ví dụ về một số bài xuất bản có lượng trích dẫn tiêu biểu cho Phenikaa Uni. Khác với các thống kê tổng phía trên, dữ liệu trích dẫn này chỉ tính cho những bài mà tác giả ghi đơn vị công tác mà hệ thống đọc hiểu là Phenikaa Uni.

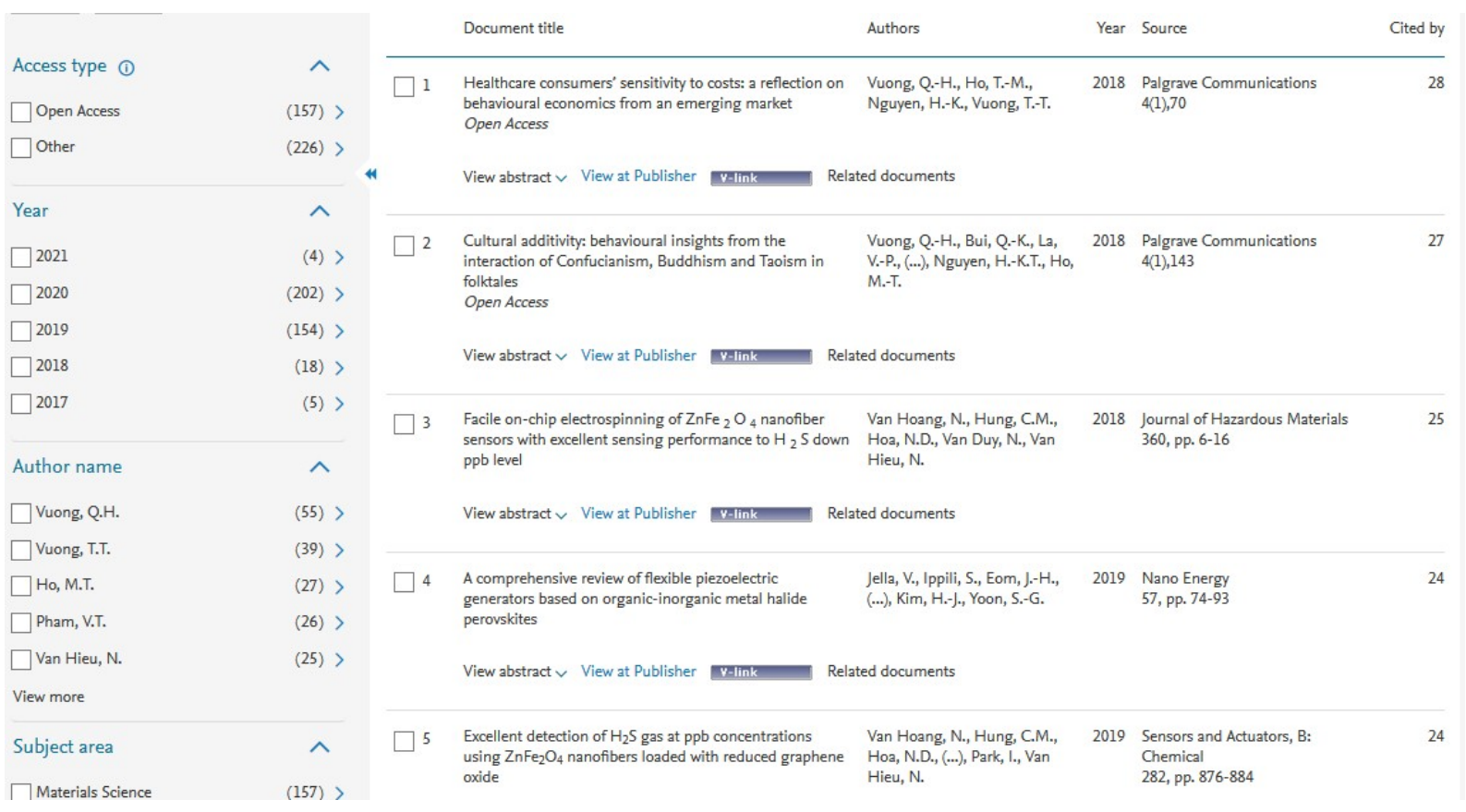

Ví dụ một số bài xuất bản cùng với số lượt trích dẫn từ các bài Scopus khác đã được thống kê

Có một vấn đề nhỏ trong lịch sử gây ra một chút tổn thất sản lượng cho Phenikaa Uni. Một số sản phẩm dưới tên "Western University, Hanoi" không được thống kê. Nhiều khả năng là các hệ thống dữ liệu lớn nhầm nó với Western University rất nổi tiếng thế giới của Ontario, Canada. Trong số các bài "thất lạc địa chi” này có bài được trích dẫn tốt như [4] (lượng trích dẫn do Scopus thống kê hiện tại là 47, và Web of Science 45).

Trên đây là một vài thống kê nhanh trích xuất từ Scopus cho thấy vài nét đại cương hoạt động khoa học của Phenikaa Uni tính đến 10-9-2020. Khi có điều kiện tìm hiểu sâu hơn, chúng tôi sẽ tiếp tục cung cấp những thông tin bổ sung cho bức tranh NCKH của đơn vị. 


\section{Tài liệu tham khảo:}

[1] Elsevier. (2020). Scopus Database. Retrieved from: https://www.scopus.com (accessed: September 10, 2020).

[2] Web of Science. (2020). Publons. Retrieved from: https://publons.com/about/home/ (accessed: September 10, 2020)

[3] Vuong, Q. H., La, V. P., Vuong, T. T., Ho, M. T., et al. (2018). An open database of productivity in Vietnam's social sciences and humanities for public use. Scientific Data, 5, 180188.

[4] Vuong, Q. H. (2018). The (ir)rational consideration of the cost of science in transition economies. Nature Human Behaviour, 2(1), 5-5. 